\title{
Correlação e associação de renda e escolaridade com condições de saúde e nutrição em obesos graves
}

\author{
Correlation and association of income and educational level \\ with health and nutritional conditions among the morbidly obese
}

Ana Paula dos Santos Rodrigues ${ }^{1}$

Erika Aparecida da Silveira ${ }^{1}$

${ }^{1}$ Programa de PósGraduação em Ciências da Saúde, Faculdade de Medicina, Universidade Federal de Goiás. 1a. Avenida s/n' ${ }^{\circ}$, Setor Leste Universitário. 74605-020 Goiânia Goiás Brasil. anapsr@gmail.com

\begin{abstract}
This article seeks to investigate the relationship between income and educational level and health and nutritional conditions among the morbidly obese. A cross-sectional study was conducted with 79 patients at first appointment, with Body Mass Index (BMI) $\geq 35 \mathrm{~kg} / \mathrm{m} 2$ and age $\geq 20$ years. The following data was collected: demographic, socioeconomic, anthropometric, lifestyle, biochemical and food intake data. Average BMI was 48.3 $\pm 6.9 \mathrm{~kg} / \mathrm{m} 2$. There was a significant negative correlation between education level and the variables of weight $(r=-0.234)$ and BMI $(r=-0.364)$ and per capita family income with daily consumption of leafy vegetables $(r=-0.263)$. After multivariate analysis, higher per capita family income was associated with the absence of heart disease (PR: 0.51, CI95\%: 0.32-0.81), higher daily consumption of leafy vegetables (PR: 1.79, CI95\%: 1.16-2.75) and candy (PR: 3.12, CI95\%: 1.21-8.04). In the morbidly obese, per capita household income was associated with absence of heart disease and higher consumption of leafy vegetables and candy. On the other hand, education level was not associated with health and nutrition conditions.

Key words Morbid obesity, Cardiovascular diseases, Food consumption, Social determinants, Vegetables
\end{abstract}

Resumo O objetivo deste artigo é investigar relações entre renda e escolaridade com condições de saúde e nutrição em obesos graves. Estudo transversal ambulatorial com 79 pacientes de primeira consulta, com Índice de Massa Corporal (IMC) $\geq 35 \mathrm{~kg} / \mathrm{m}^{2}$ e idade $\geq 20$ anos. Coletaram-se dados: sociodemográficos, antropométricos, estilo de vida, exames bioquimicos e consumo alimentar. $O$ IMC médio foi $48,3 \pm 6,9 \mathrm{~kg} / \mathrm{m}^{2}$. Observou-se correlação negativa significante de escolaridade com variáveis peso $(r=-0,234)$ e IMC $(r=-0,364) e$ de renda familiar per capita com consumo diário de vegetal A $(r=-0,263)$. Após análise multivariada maior renda familiar per capita se associou à ausência de cardiopatia (RP: 0,51, IC95\%: 0,32-0,81), maior consumo diário de vegetal $A$ (RP: 1,79, IC95\%: 1,16-2,75) e doces (RP: 3,12, IC95\%: 1,21-8,04). Em obesos graves a maior renda familiar per capita se associou à ausência de cardiopatia e maior consumo de vegetais folhosos e doces. Já a escolaridade não se manteve associada às condições de saúde e nutrição.

Palavras-chave Obesidade grave, Doenças cardiovasculares, Consumo de alimentos, Determinantes sociais, Verduras 


\section{Introdução}

A obesidade é uma doença epidêmica no mun$\mathrm{do}^{1,2}$. Na população adulta a prevalência chega a $33,8 \%$ nos Estados Unidos ${ }^{3}$ e atinge $14,8 \%$ no Brasil $^{4}$. A alta prevalência da obesidade é preocupante, pois está relacionada à maior mortalidade $\mathrm{e}$ incidência de doenças cardiovasculares, hipertensão, diabetes, dislipidemia, cálculo biliar, osteoartrite, apneia do sono e certos tipos de câncer ${ }^{2,5,6}$.

A obesidade tem origem em uma interação complexa entre fatores biológicos, genéticos, ambientais, sociais e psicológicos, que levam a um balanço energético positivo e, consequentemente, ao ganho de peso $^{2}$. Dentre estes fatores, alguns estudos apontam associação entre obesidade e aspectos socioeconômicos como renda e escolaridade $^{7-9}$. Algumas pesquisas demonstram que associação inversa entre escolaridade e obesidade, principalmente entre as mulheres ${ }^{7-9}$. Estudo nas regiões Nordeste e Sudeste do Brasil observou associação entre renda e obesidade em homens". No entanto, essas pesquisas avaliaram indivíduos com sobrepeso e obesidade, sendo importante conhecer a relação de renda e escolaridade com as condições de saúde e nutrição em obesos graves, ou seja, com IMC $\geq 35 \mathrm{~kg} / \mathrm{m}^{2}$. Aspectos relacionados aos determinantes sociais de saúde como renda e escolaridade merecem ser investigados em obesos graves, bem como com as condições de saúde e nutrição desse grupo populacional.

O tratamento clínico da obesidade grave tem se mostrado pouco efetivo em manter a redução de peso ${ }^{10}$, assim compreender as inter-relações entre renda e escolaridade com variáveis de saúde e nutrição podem auxiliar na maior compreensão do cenário e das condições ambientais em que esses pacientes estão inseridos e a partir desse conhecimento, elaborar intervenções mais específicas e adequadas ao perfil dessa população. Ademais, estudos com obesos graves quase sempre abordam questões do período pós-operatório de cirurgia bariátrica, sendo poucos estudos buscando compreender a situação desses pacientes em seu contexto social ${ }^{11-13}$. Diante disso, o objetivo deste estudo foi investigar as relações de renda e escolaridade conforme as condições de saúde e nutrição em obesos graves.

\section{Métodos}

Realizou-se estudo transversal com obesos graves que buscaram atendimento no Ambulatório de Nutrição e Obesidade Grave do Hospital das
Clínicas (HC) da Universidade Federal de Goiás (UFG), Goiânia-GO. Esse ambulatório desenvolve atividades de pesquisa, ensino e extensão, prestando atendimento exclusivamente a obesos com IMC $\geq 35 \mathrm{~kg} / \mathrm{m}^{2}$ desde 2007 . A população do estudo foi constituída por todos os pacientes de primeira consulta no ambulatório de outubro de 2007 a outubro de 2009, excluindo-se cadeirantes. Os pacientes atendidos apresentavam como critério de inclusão: idade $\geq 20$ anos e Índice de Massa Corporal (IMC) $\geq 35 \mathrm{~kg} / \mathrm{m}^{2}$, ou seja, obesidade grave (graus II e III).

Toda equipe de profissionais do ambulatório recebeu treinamento prévio para a coleta de dados da pesquisa e para o atendimento dos pacientes conforme protocolo nutricional especializado e individualizado ${ }^{14}$. A aferição do peso e altura foram realizados por Nutricionistas ou acadêmicos de Nutrição após treinamento e padronização visando reprodutibilidade e validade da informação inter e intra-antropometrista ${ }^{15}$.

As variáveis estudadas e coletadas em formulário padronizado e pré-testado no momento da primeira consulta foram: demográficas - sexo, idade, estado civil, cor da pele; socioeconômicas - escolaridade em anos de estudo e renda familiar per capita; condições de saúde - hipertensão, diabetes, dislipidemia, cardiopatia, litíase biliar, outras doenças; glicemia de jejum, lipidograma (colesterol total, HDL colesterol, LDL colesterol, triglicérides) e ácido úrico; estilo de vida tabagismo, ingestão de bebida alcoólica, prática de atividade física; condição nutricional - peso, altura e grau de obesidade pelo Índice de Massa Corporal (IMC) e consumo de alimentos saudáveis e de risco avaliado por meio de frequência de consumo alimentar ${ }^{16}$.

A variável escolaridade foi categorizada em 0-8 anos e $\geq 9$ anos de estudo e a renda familiar per capita foi categorizada em $1^{\circ}$ e $2^{\circ}$ quartis de renda e $3^{\circ}$ e $4^{\circ}$ quartis de renda.

Os exames bioquímicos foram solicitados na primeira consulta e os resultados anotados no primeiro retorno do paciente ao ambulatório em um intervalo de até 30 dias. Até esse retorno o paciente ainda se encontrava em fase de avaliação diagnóstica e sem receber nenhuma intervenção que pudesse modificar os resultados.

Considerou-se como prática de atividade física a realização de no mínimo 150 minutos semanais de atividade aeróbia de moderada a intensa, conforme recomendação da Organização Mundial da Saúde ${ }^{17}$.

O peso foi aferido utilizando-se balança tipo plataforma da marca Welmy, capacidade até 300 
$\mathrm{kg}$, escala de $100 \mathrm{~g}$, com os participantes vestindo roupas leves e descalços, posicionando-se de costas para a escala, no centro da plataforma da balança e distribuindo o peso entre ambos os pés, conforme técnica proposta pela $\mathrm{WHO}^{18}$. A altura foi medida utilizando antropômetro vertical milimetrado da balança tipo plataforma, com escala de $0,1 \mathrm{~cm}$, estando os indivíduos em posição ereta, a cabeça posicionada de forma que a visão forme linha perpendicular ao corpo, braços estendidos ao longo do corpo e descalços ${ }^{18}$.

Dividiu-se o peso $(\mathrm{kg})$ pela altura $(\mathrm{m})$ elevada ao quadrado para calcular o IMC e foram utilizados os seguintes pontos de corte: 35,0 a 39,9 $\mathrm{kg} / \mathrm{m}^{2}$ obesidade grau II; 40,0 a 49,9 kg/m² obesidade grau III; 50,0 a $59,9 \mathrm{~kg} / \mathrm{m}^{2}$ superobesidade e $\geq 60,0 \mathrm{~kg} / \mathrm{m}^{2}$ super/superobesidade ${ }^{19}$.

A análise do consumo alimentar foi feita utilizando-se Questionário de Frequência de Consumo (QFC) adaptado de Block et al. ${ }^{16}$ sendo que os alimentos foram divididos em 2 grupos: alimentos saudáveis - leguminosas, leite e derivados, frutas, vegetal A (vegetais com 1 a 7,5\% de carboidratos, como alface, couve, acelga, mostarda, etc.) e vegetal B (vegetais com 7,5 a 10,5\% de carboidratos, como cenoura, beterraba, vagem, etc.); e alimentos de risco - quitandas e salgados, açúcar, doces, frituras e refrigerante. $\mathrm{O}$ consumo dos alimentos foi categorizado em consumo diário "sim" e "não" e ainda analisado na sua forma contínua.

Quanto aos exames laboratoriais, foi utilizado como valor de referência para a glicemia o critério da American Diabetes Association ${ }^{20}$, sendo normal glicemia de jejum $<100 \mathrm{mg} / \mathrm{dL}$, para o perfil lipídico utilizou-se como referência o critério da Sociedade Brasileira de Cardiologia ${ }^{21}$, sendo considerado normal colesterol total $<200 \mathrm{mg} /$ dL, LDL-c $<160 \mathrm{mg} / \mathrm{dL}, \mathrm{HDL}-\mathrm{c}>50 \mathrm{mg} / \mathrm{dL}$ para mulheres e $>40 \mathrm{mg} / \mathrm{dL}$ para homens e triglicérides $<150 \mathrm{mg} / \mathrm{dL}$. Para o ácido úrico considerouse normal valores entre 2,6 e 6,0 para mulheres e 3,5 e 7,2 para homens ${ }^{22}$.

A análise estatística foi feita no programa Stata 8.0. Para variáveis categóricas utilizou-se frequência absoluta e relativa e para variáveis contínuas, média e desvio-padrão. Para comparação entre as médias de IMC foi utilizado test t de Student. Analisou-se a correlação da escolaridade e renda familiar per capita com as variáveis antropométricas, de consumo alimentar, prática de atividade física e exames bioquímicos utilizando-se o teste de correlação de Pearson ou Spearman, após avaliar a normalidade pelo teste de Shapiro-Wilk. Para verificar a associação das variáveis de condições de saúde e nutrição com renda familiar per capita e escolaridade utilizouse o teste do Qui-quadrado ou exato de Fisher. Foi considerado estatisticamente significante o valor $\mathrm{p} \leq 0,05$. Calculou-se ainda por regressão de Poisson simples a razão de prevalência e intervalo de confiança de $95 \%$. As variáveis com valor $\mathrm{p} \leq 0,20$ foram levadas para análise multivariada por regressão de Poisson, permanecendo no modelo final apenas as variáveis com valor $\mathrm{p} \leq 0,05$.

Este estudo está inserido em um projeto maior denominado "Evolução Nutricional e Clínica de Pacientes Candidatos a Cirurgia Bariátrica em Período Pré e Pós-Operatório", o qual foi aprovado pelo Comitê de Ética em Pesquisa Médica, Humana e Animal do Hospital das Clínicas da Universidade Federal de Goiás (CEPMHA/ HC-UFG).

\section{Resultados}

A média de IMC foi de $48,3 \pm 6,9 \mathrm{~kg} / \mathrm{m}^{2}$. A categoria de IMC mais prevalente foi de 40 a $49,9 \mathrm{~kg} /$ $\mathrm{m}^{2}$ com $53,8 \%$, seguida por 50 a $59,9 \mathrm{~kg} / \mathrm{m}^{2} \mathrm{com}$ $28,2 \%, 35,0$ a $39,9 \mathrm{~kg} / \mathrm{m}^{2}$ com $10,3 \%$ e $\geq 60,0 \mathrm{~kg} /$ $\mathrm{m}^{2} \operatorname{com} 7,7 \%$

Quanto às características socioeconômicas e demográficas dos 79 pacientes dessa pesquisa, $91,1 \%$ eram mulheres, $60,7 \%$ possuíam entre 30 e 49 anos de idade, 64,6\% eram casados/união estável e 35,4\% tinham de 5 a 8 anos de estudo. Quanto à renda, 54,6\% dos pacientes possuíam renda própria, sendo a média $\mathrm{R} \$ 531,90 \pm 403,95$ e a renda familiar per capita $\mathrm{R} \$ 278,05 \pm 191,22$. Mais de $75 \%$ dos pacientes possuíam renda familiar per capita menor do que 1 salário mínimo (Tabela 1).

Observa-se que os níveis médios de glicemia de jejum e triglicérides estiveram acima dos valores de referência (Tabela 1), sendo que $42 \%$ apresentaram glicemia acima do valor de normalidade e 38,8\% apresentaram hipertrigliceridemia. A prevalência de hipercolesterolemia foi de 38,8\%, HDL baixo 75,8\%, LDL elevado 6,2\% e $34,0 \%$ com ácido úrico acima dos valores de normalidade (dados não apresentados em tabela).

Na análise de correlação (Tabela 2), observase correlação negativa de escolaridade com IMC $(\mathrm{p}=0,001, \mathrm{r}=-0,364)$ e peso $(\mathrm{p}=0,039$ e $\mathrm{r}=$ $-0,234)$, porém os valores de $\mathrm{r}$ indicam correlação fraca. Também foi observada correlação negativa de renda familiar per capita com consumo diário de vegetal A ( $\mathrm{p}=0,024$ e $\mathrm{r}=-0,263)$.

$\mathrm{Na}$ análise de associação, a escolaridade esteve 


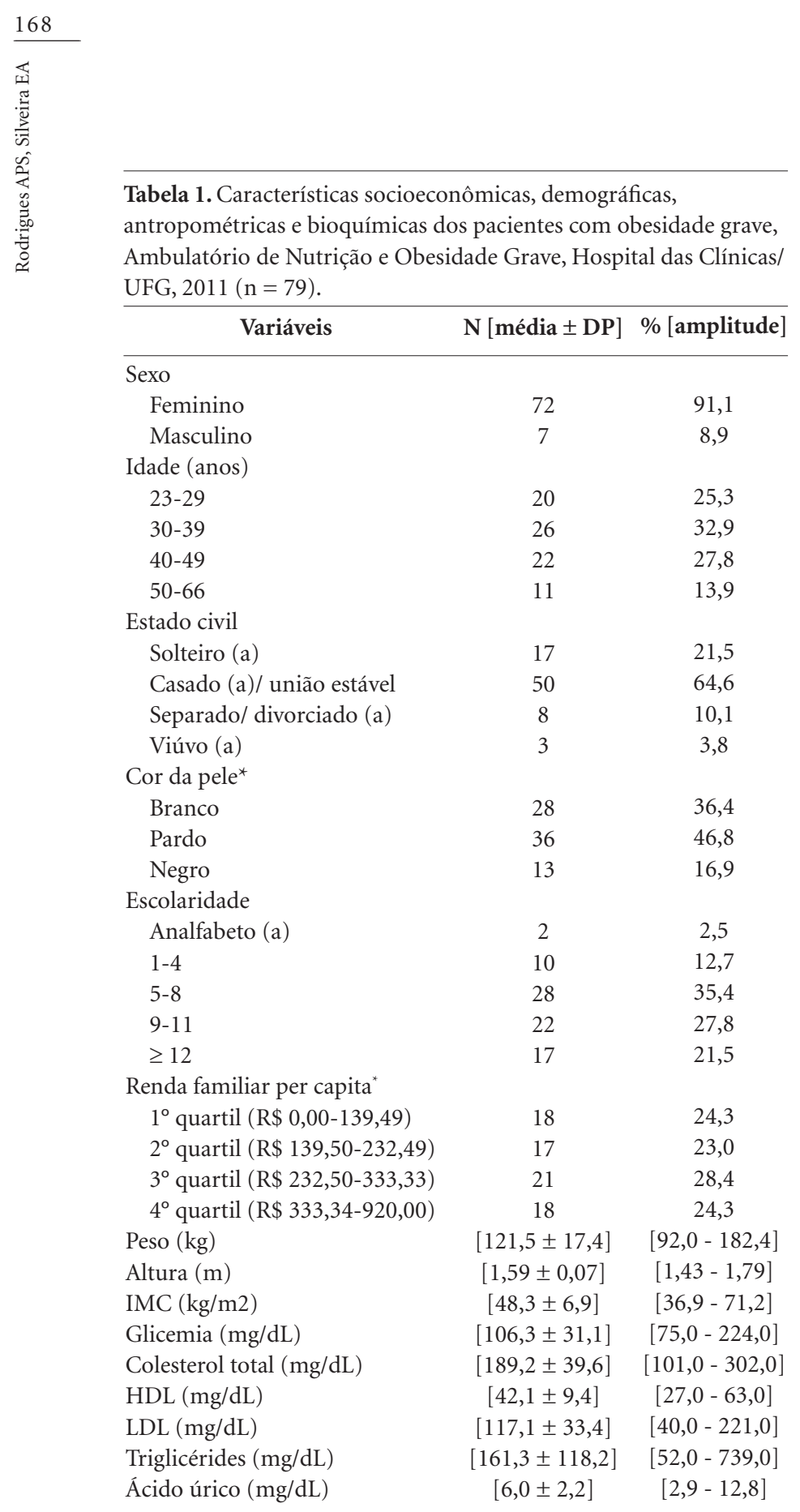

Cor da pele: $\mathrm{n}=77$; renda familiar per capita: $\mathrm{n}=74$.

associada à hipertensão arterial $(\mathrm{p}=0,009) \mathrm{com}$ maior prevalência para indivíduos de menor escolaridade (Tabela 3). Para a renda familiar per capita (Tabela 4) observou-se associação com: presença de cardiopatia $(\mathrm{p}=0,046)$, consumo diário de vegetal $\mathrm{A}(\mathrm{p}=0,008)$ e consumo diário de doces $(\mathrm{p}=0,027)$. O sentido dessas associações variou conforme a seguir: renda foi significativamente maior naqueles que não apresentaram cardiopatia; consumo diário de vegetal A e doces foram maiores naqueles com maior renda familiar per capita.

Após análise de regressão de Poisson multivariada, observa-se que a hipertensão arterial não se manteve associada à escolaridade e para renda familiar per capita, as variáveis presença de cardiopatia, consumo diário de vegetal A e consumo diário de doces mantiveram a associação (Tabela 5).

\section{Discussão}

Notou-se predomínio de mulheres, sendo o mesmo observado em outros estudos ${ }^{11,23-28}$, o que pode ser explicado pela maior utilização dos serviços de saúde pelo sexo feminino ${ }^{29}$ ou pela maior prevalência de obesidade mórbida entre as mulheres ${ }^{3,4}$. Os dados apontaram também concentração de idade na faixa de 30 a 49 anos, o que está de acordo com outra pesquisa em que a prevalência de obesidade grave reduz a partir dos 55 anos $^{13}$. Houve concentração de pacientes das classes de menor escolaridade e menor renda, achado similar a outros estudos com pacientes de ambulatórios de Hospitais Escola ${ }^{24,25}$.

Os resultados dos exames bioquímicos evidenciaram alta prevalência de alterações metabólicas nos obesos graves. A prevalência de glicemia acima do normal $(42,0 \%)$ foi maior do que a de diabetes melito (13,9\%), podendo indicar início de intolerância à glicose ou falta de controle do diabetes melito. A prevalência de hipercolesterolemia e hipertrigliceridemia neste estudo foram superiores à metanálise de Buchwald et al. ${ }^{26}$, sendo o mesmo observado para o ácido úrico em relação ao estudo de Porto et al. ${ }^{23}$.

Nesta pesquisa, houve correlação negativa significante de escolaridade com IMC e peso, ou seja, quanto maior o peso e o IMC menor a escolaridade. Outros estudos mostram relação inversa entre escolaridade e obesidade $e^{9,27,30,31}$, sendo o mesmo encontrado nessa pesquisa para a obesidade grave. Na análise de associação, notase maior prevalência de hipertensão arterial em indivíduos de menor escolaridade. Associação inversa entre nível educacional e pressão arterial foi apontada em outros estudos, corroborando os achados desta pesquisa ${ }^{32,33}$.

Já na análise da renda familiar per capita houve correlação inversa com o consumo diário de vegetal A, apesar de fraca $(r=-0,263)$. Após analise por regressão múltipla, observa-se que os obesos graves de menor renda têm 1,79 mais chance de não consumir vegetal A diariamente do que os de maior renda. 
Tabela 2. Correlação entre escolaridade e renda familiar per capita com variáveis antropométricas, consumo alimentar, prática de atividade física e exames bioquímicos dos pacientes obesos graves, Ambulatório de Nutrição e Obesidade Grave, Hospital das Clínicas/UFG, 2011.

\begin{tabular}{|c|c|c|}
\hline Variáveis & $\begin{array}{l}\text { Escolaridade } \\
\text { r (valor-p) }\end{array}$ & $\begin{array}{c}\text { Renda familiar per capita } \\
\text { r (valor-p) }\end{array}$ \\
\hline \multicolumn{3}{|l|}{ Antropométricas } \\
\hline Peso & $-0,234(0,039)^{*}$ & $-0,022(0,854)^{* *}$ \\
\hline Altura & $0,175(0,124)^{*}$ & $0,068(0,567)^{*}$ \\
\hline IMC & $-0,364(0,001)^{*}$ & $-0,003(0,979)^{* *}$ \\
\hline \multicolumn{3}{|l|}{ Alimentos saudáveis ${ }^{\#}$} \\
\hline Leguminosas & $-0,021(0,860)^{*}$ & $0,028(0,816)^{* *}$ \\
\hline Leite e derivados & $-0,162(0,162)^{*}$ & $0,012(0,918)^{* *}$ \\
\hline Frutas & $-0,216(0,061)^{*}$ & $-0,186(0,116)^{* *}$ \\
\hline Vegetal A & $0,109(0,347)^{*}$ & $-0,263(0,024)^{* *}$ \\
\hline Vegetal B & $0,013(0,914)^{*}$ & $0,050(0,676)^{* *}$ \\
\hline \multicolumn{3}{|l|}{ Alimentos de risco ${ }^{\#}$} \\
\hline Quitandas e salgados & $-0,097(0,404)^{*}$ & $0,017(0,888)^{*}$ \\
\hline Açúcar & $0,122(0,295)^{*}$ & $0,049(0,683)^{* *}$ \\
\hline Doces & $-0,154(0,186)^{*}$ & $-0,028(0,815)^{*}$ \\
\hline Frituras & $-0,038(0,742)^{*}$ & $0,072(0,544)^{* *}$ \\
\hline Refrigerante & $0,154(0,185)^{*}$ & $-0,142(0,229)^{* *}$ \\
\hline \multicolumn{3}{|l|}{ Prática de atividade física } \\
\hline Minutos diários & $0,073(0,740)^{*}$ & $0,079(0,725)^{*}$ \\
\hline \multicolumn{3}{|l|}{ Exames bioquímicos } \\
\hline Glicemia de jejum & $0,042(0,730)^{*}$ & $-0,053(0,675)^{* *}$ \\
\hline Colesterol total & $-0,036(0,770)^{*}$ & $0,004(0,973)^{*}$ \\
\hline HDL & $-0,006(0,964)^{*}$ & $0,132(0,300)^{* *}$ \\
\hline LDL & $-0,008(0,948)^{*}$ & $-0,046(0,720)^{*}$ \\
\hline Triglicérides & $0,004(0,974)^{*}$ & $-0,029(0,821)^{* *}$ \\
\hline Ácido úrico & $-0,232(0,112)^{*}$ & $-0,216(0,149)^{* *}$ \\
\hline
\end{tabular}

"Correlação de Pearson; ${ }^{* *}$ Correlação de Spearman; ${ }^{*}$ Consumo diário.

Tabela 3. Condições de saúde e nutrição em pacientes obesos graves conforme nível de escolaridade com respectiva razão de prevalência e teste de associação. Ambulatório de Nutrição e Obesidade Grave, Hospital das Clínicas/UFG, $2011(\mathrm{n}=79)$.

\begin{tabular}{|c|c|c|c|c|c|}
\hline \multirow[b]{2}{*}{ Variáveis } & \multirow[b]{2}{*}{$\mathbf{N}(\%)$} & \multicolumn{2}{|c|}{ Escolaridade $(\mathrm{N}=79)$} & \multirow[b]{2}{*}{ RP (IC) } & \multirow[b]{2}{*}{ Valor $\mathbf{p}^{*}$} \\
\hline & & $\begin{array}{c}0 \text { a } 8 \\
\text { N (\%) }\end{array}$ & $\begin{array}{c}\geq 9 \\
\mathrm{~N}(\%)\end{array}$ & & \\
\hline Hipertensão & & & & & 0,009 \\
\hline Sim & $46(58,20)$ & $29(63,04)$ & $17(36,96)$ & $1,89(1,11-3,22)$ & \\
\hline Não & $33(41,77)$ & $11(33,33)$ & $22(66,67)$ & 1 & \\
\hline $\begin{array}{l}\text { Dislipidemia } \\
\quad \text { Sim }\end{array}$ & $\begin{array}{l}35(44,30) \\
44(55,70)\end{array}$ & $\begin{array}{l}20(57,14) \\
20(45,45)\end{array}$ & $\begin{array}{l}15(42,86) \\
24(54,55)\end{array}$ & $\begin{array}{c}1,26(0,81-1,94) \\
1\end{array}$ & 0,302 \\
\hline Não & & & & & $0,755^{* *}$ \\
\hline $\begin{array}{c}\text { Diabetes } \\
\text { Sim } \\
\text { Não }\end{array}$ & $\begin{array}{c}11(13,9) \\
68(86,08)\end{array}$ & $\begin{array}{c}5(45,45) \\
35(51,47)\end{array}$ & $\begin{array}{c}6(54,55) \\
33(48,53)\end{array}$ & $\begin{array}{c}1 \\
1,13(0,57-2,26)\end{array}$ & 0,70 \\
\hline $\begin{array}{l}\text { Litíase biliar } \\
\text { Sim } \\
\text { Não }\end{array}$ & $\begin{array}{c}9(11,4) \\
70(88,61)\end{array}$ & $\begin{array}{c}7(77,78) \\
33(47,14)\end{array}$ & $\begin{array}{c}2(22,22) \\
37(52,86)\end{array}$ & $\begin{array}{c}1,67(1,07-2,54) \\
1\end{array}$ & $0,154^{* *}$ \\
\hline
\end{tabular}


Tabela 3. continuação

\begin{tabular}{|c|c|c|c|c|c|}
\hline \multirow[b]{2}{*}{ Variáveis } & \multirow[b]{2}{*}{$\mathrm{N}(\%)$} & \multicolumn{2}{|c|}{ Escolaridade $(\mathrm{N}=79)$} & \multirow[b]{2}{*}{ RP (IC) } & \multirow[b]{2}{*}{ Valor $\mathbf{p}^{*}$} \\
\hline & & $\begin{array}{c}0 \text { a } 8 \\
\text { N (\%) }\end{array}$ & $\begin{array}{c}\geq 9 \\
\mathrm{~N}(\%)\end{array}$ & & \\
\hline Cardiopatia & & & & & $1,000^{* *}$ \\
\hline Sim & $4(5,1)$ & $2(50,00)$ & $2(50,00)$ & 1 & \\
\hline Não & $75(94,94)$ & $38(50,67)$ & $37(49,33)$ & $1,01(0,37-2,79)$ & \\
\hline Outras doenças & & & & & 0,205 \\
\hline Sim & $27(34,2)$ & $11(40,74)$ & $16(59,26)$ & 1 & \\
\hline Não & $52(65,82)$ & $29(55,77)$ & $23(44,23)$ & $1,37(0,82-2,30)$ & \\
\hline Tabagismo & & & & & $0,484^{* *}$ \\
\hline Não fumante & $56(70,9)$ & $26(46,43)$ & $30(53,57)$ & 1 & \\
\hline Ex-fumante & $17(21,5)$ & $10(58,82)$ & $7(41,18)$ & $1,27(0,78-2,07)$ & \\
\hline Fumante & $6(7,6)$ & $4(66,67)$ & $2(33,33)$ & $1,44(0,76-2,71)$ & \\
\hline Ingestão de bebida alcoólica & & & & & $0,083^{* *}$ \\
\hline Sim & $14(17,7)$ & $4(28,57)$ & $10(71,43)$ & 1 & \\
\hline Não & $65(82,3)$ & $36(55,38)$ & $29(44,62)$ & $1,94(0,82-4,59)$ & \\
\hline Prática de atividade física & & & & & 0,415 \\
\hline Sim & $23(29,1)$ & $10(43,48)$ & $13(56,52)$ & 1 & \\
\hline Não & $56(70,9)$ & $30(53,57)$ & $26(46,43)$ & $1,23(0,73-2,09)$ & \\
\hline \multicolumn{6}{|l|}{ Alimentos saudáveis\# } \\
\hline Leguminosas & & & & & 0,727 \\
\hline Sim & $47(61,84)$ & $24(51,06)$ & $23(48,94)$ & 1 & \\
\hline Não & $29(38,16)$ & $16(55,17)$ & $13(44,83)$ & $1,08(0,70-1,67)$ & \\
\hline Leite e derivados & & & & & 0,679 \\
\hline Sim & $34(44,74)$ & $17(50,00)$ & $17(50,00)$ & 1 & \\
\hline Não & $42(55,26)$ & $23(54,76)$ & $19(45,24)$ & $1,10(0,71-1,70)$ & \\
\hline Frutas & & & & & 0,075 \\
\hline Sim & $28(36,84)$ & $11(39,29)$ & $17(60,71)$ & 1 & \\
\hline Não & $48(63,16)$ & $29(60,42)$ & $19(39,58)$ & $1,54(0,92-2,58)$ & \\
\hline Vegetal A & & & & & 0,262 \\
\hline Sim & $50(65,79)$ & $24(48,00)$ & $26(52,00)$ & 1 & \\
\hline Não & $26(34,21)$ & $16(61,54)$ & $10(38,46)$ & $1,28(0,84-1,96)$ & \\
\hline Vegetal B & & & & & 0,408 \\
\hline Sim & $28(36,84)$ & $13(46,43)$ & $15(53,57)$ & $1,21(0,76-1,94)$ & \\
\hline Não & $48(63,16)$ & $27(56,25)$ & $21(43,75)$ & 1 & \\
\hline \multicolumn{6}{|l|}{ Alimentos de risco\# } \\
\hline Quitandas e salgados & & & & & $0,747^{* *}$ \\
\hline Sim & $11(14,47)$ & $5(45,45)$ & $6(54,55)$ & 1 & \\
\hline Não & $65(85,53)$ & $35(53,85)$ & $30(46,15)$ & $1,18(0,59-2,36)$ & \\
\hline Açúcar & & & & & 0,550 \\
\hline Sim & $47(61,84)$ & $26(55,32)$ & $21(44,68)$ & $1,15(0,72-1,81)$ & \\
\hline Não & $29(38,16)$ & $14(48,28)$ & $15(51,72)$ & 1 & \\
\hline Doces & & & & & $0,702^{* *}$ \\
\hline Sim & $7(9,21)$ & $3(42,86)$ & $4(57,14)$ & 1 & \\
\hline Não & $69(90,79)$ & $37(53,62)$ & $32(46,38)$ & $1,25(0,51-3,04)$ & \\
\hline Frituras & & & & & $0,127^{* *}$ \\
\hline Sim & $13(17,11)$ & $4(30,77)$ & $9(69,23)$ & 1 & \\
\hline Não & $63(82,89)$ & $36(57,14)$ & $27(42,86)$ & $1,86(0,80-4,34)$ & \\
\hline Refrigerante & & & & & 0,242 \\
\hline Sim & $24(31,58)$ & $15(62,50)$ & $9(37,50)$ & $1,30(0,85-1,98)$ & \\
\hline Não & $52(68,42)$ & $25(48,08)$ & $27(51,92)$ & 1 & \\
\hline
\end{tabular}

"Teste do Qui-quadrado; * Teste exato de Fischer; ${ }^{*}$ Consumo diário. RP = razão de prevalência, IC = intervalo de confiança de 95\% 
Tabela 4. Condições de saúde e nutrição em pacientes obesos graves conforme nível de renda com respectiva razão de prevalência e teste de associação. Ambulatório de Nutrição e Obesidade Grave, Hospital das Clínicas/ UFG, $2011(\mathrm{n}=79)$.

\begin{tabular}{|c|c|c|c|c|c|}
\hline \multirow[b]{2}{*}{ Variáveis } & \multirow[b]{2}{*}{ N (\%) } & \multicolumn{2}{|c|}{$\begin{array}{l}\text { Renda familiar per capita } \\
\qquad(\mathrm{N}=74)\end{array}$} & \multirow[b]{2}{*}{ RP (IC) } & \multirow[b]{2}{*}{ Valor $\mathrm{p}$} \\
\hline & & $\begin{array}{c}1^{\circ} \text { e } 2^{\circ} \text { quartis } \\
\mathrm{N}(\%)\end{array}$ & $\begin{array}{c}3^{\circ} \text { e } 4^{\circ} \text { quartis } \\
\mathrm{N}(\%)\end{array}$ & & \\
\hline Hipertensão & & & & & 0,052 \\
\hline Sim & $42(56,76)$ & $24(57,14)$ & $18(42,86)$ & $1,66(0,96-2,88)$ & \\
\hline Não & $32(43,24)$ & $11(34,38)$ & $21(65,63)$ & 1 & \\
\hline Dislipidemia & & & & & 0,209 \\
\hline Sim & $31(41,89)$ & $12(38,71)$ & $19(61,29)$ & 1 & \\
\hline Não & $43(58,11)$ & $23(53,59)$ & $20(46,51)$ & $1,38(0,82-2,34)$ & \\
\hline Diabetes & & & & & $1,000^{* *}$ \\
\hline Sim & $9(12,16)$ & $4(44,44)$ & $5(55,56)$ & 1 & \\
\hline Não & $65(87,84)$ & $31(47,69)$ & $34(52,31)$ & $1,07(0,49-2,34)$ & \\
\hline Litíase biliar & & & & & $0,701^{* *}$ \\
\hline Sim & $7(9,46)$ & $4(57,14)$ & $3(42,86)$ & $1,24(0,62-2,48)$ & \\
\hline Não & $67(90,54)$ & $31(46,27)$ & $36(53,73)$ & 1 & \\
\hline $\begin{array}{l}\text { Cardiopatia } \\
\text { Sim }\end{array}$ & $4(5,41)$ & $4(100,00)$ & $0(0,00)$ & $2,26(1,73-2,94)$ & $0,046^{* *}$ \\
\hline Não & $70(94,59)$ & $31(44,29)$ & $39(55,71)$ & 1 & 0260 \\
\hline Outras doenças & $25(33,78)$ & $10(40,00)$ & $15(60,00)$ & 1 & 0,509 \\
\hline Sim & $49(66,22)$ & $25(51,02)$ & $24(48,98)$ & $1,28(0,73-2,23)$ & \\
\hline Tabagismo & & & & & $0,569^{* *}$ \\
\hline Não fumante & $53(71,62)$ & $23(43,40)$ & $30(56,60)$ & 1 & \\
\hline Ex-fumante & $16(21,62)$ & $9(56,25)$ & $7(43,75)$ & $1,30(0,76-2,21)$ & \\
\hline Fumante & $5(6,76)$ & $3(60,00)$ & $2(40,00)$ & $1,38(0,63-3,03)$ & \\
\hline $\begin{array}{l}\text { Ingestão de bebida alcoólica } \\
\text { Sim }\end{array}$ & $13(17,57)$ & $5(38,46)$ & $8(61,54)$ & 1 & 0,482 \\
\hline Não & $61(82,43)$ & $30(49,18)$ & $31(50,82)$ & $1,28(0,61-2,68)$ & \\
\hline Prática de atividade física & $22(29,73)$ & $7(31,82)$ & $15(68,18)$ & 1 & 0,083 \\
\hline $\begin{array}{l}\text { Sim } \\
\text { Não }\end{array}$ & $52(70,27)$ & $28(53,85)$ & $24(46,15)$ & $1,69(0,87-3,29)$ & \\
\hline
\end{tabular}

Vale destacar na presente pesquisa, uma importante associação e correlação entre a renda e o consumo de vegetais folhosos, assim indivíduos de menor renda possuem menor probabilidade de consumir esses alimentos, principalmente considerando que são tão importantes numa dieta de redução calórica e para uma alimentação saudável.

Não foi demonstrada correlação e associação entre renda familiar per capita e variáveis antropométricas, como peso e IMC, nos pacientes com obesidade grave. Esses achados diferem da população em geral onde pesquisas já mostraram associação de renda com obesidade ${ }^{9,13}$.

Menor prevalência de cardiopatia esteve associada ao maior nível de renda familiar per ca- pita, assim evidencia-se que maior renda protege contra o risco de cardiopatia em obesos graves. Similar a esses achados, algumas pesquisas indicaram que a prevalência e o risco para doença cardiovascular é maior em indivíduos com menor nível socioeconômico ${ }^{34,35}$.

Nos obesos graves o consumo diário de vegetal A foi significativamente maior para os indivíduos de maior renda, sendo o mesmo verificado para o consumo diário de doces. Na POF 2002-2003 ${ }^{36}$ o consumo de verduras e legumes aumentou de maneira uniforme conforme aumento do nível de rendimentos familiares. Enquanto na POF 2002$2003^{36}$ foi observado que as classes de rendimentos superiores consomem 50\% menos açúcar do que as classes mais baixas, ou seja, diferente do 


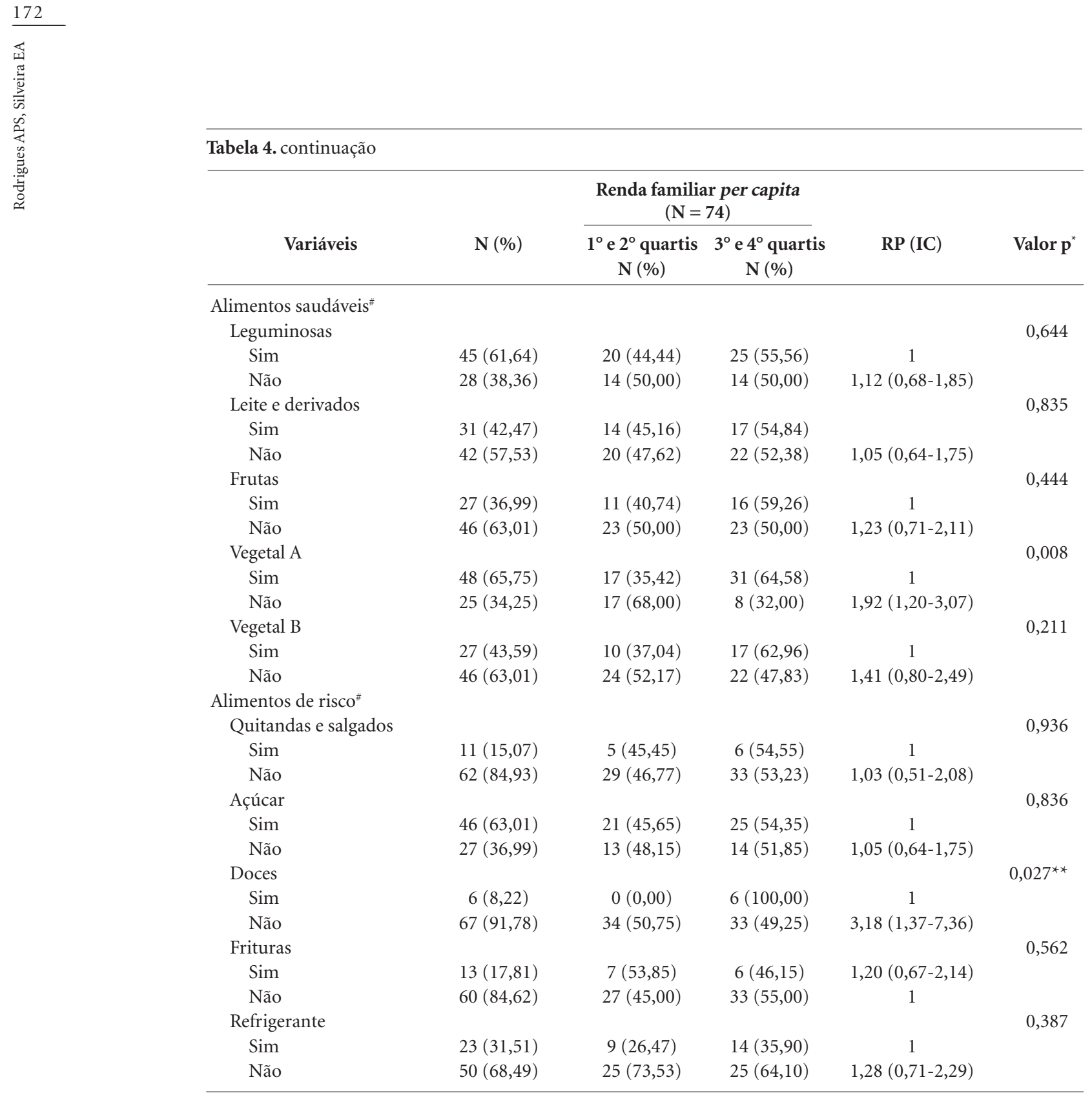

* Teste do Qui-quadrado; ${ }^{* *}$ Teste exato de Fischer; ${ }^{*}$ Consumo diário. RP = razão de prevalência, IC = intervalo de confiança de 95\%

Tabela 5. Regressão multivariada das condições de saúde e nutrição conforme nível de escolaridade e renda em obesos graves, Ambulatório de Nutrição e Obesidade Grave, Hospital das Clínicas/UFG, 2011 (n = 79).

\begin{tabular}{lcc}
\hline \multicolumn{1}{c}{ Variáveis } & RP (IC95\%) & ${\text { Valor } \mathbf{p}^{*}}^{*}$ \\
\hline Hipertensão (sim) & Escolaridade & \\
Litíase biliar (sim) & $1,46(0,87-2,46)$ & 0,155 \\
Ingestão de bebida alcoólica (não) & $1,62(0,96-2,74)$ & 0,070 \\
Consumo diário de frutas (não) & $2,01(0,81-4,98)$ & 0,132 \\
Consumo diário de frituras (não) & $1,91(0,85-4,31)$ & 0,119 \\
& $1,35(0,87-2,08)$ & 0,177 \\
Hipertensão (sim) & Renda familiar per capita & 0,129 \\
Cardiopatia (sim) & $1,52(0,89-2,60)$ & 0,413 \\
Prática de atividade física (não) & $0,51(0,32-0,81)$ & 0,009 \\
Consumo diário de vegetal A (não) & $1,33(0,67-2,64)$ & $<0,001$ \\
Consumo diário de doces (não) & $1,79(1,16-2,75)$ & \\
\hline
\end{tabular}

"Estatística Wald RP = razão de prevalência IC95\% = intervalo de confiança de 95\%. 
presente estudo. Já neste estudo não foi encontrada diferença entre os grupos para o consumo de açúcar, entretanto, o consumo de doces foi significativamente maior para o grupo de maior renda, podendo ser uma característica específica da população de obesos graves. Assim, sugere-se que outras pesquisas avaliem o consumo alimentar de indivíduos com obesidade grave para que outras peculiaridades sejam identificadas.

Entre as possíveis limitações metodológicas desta pesquisa destaca-se a uniformidade da população estudada quanto ao nível de renda, em sua maioria de baixo poder aquisitivo, o que pode ter reduzido a possibilidade de encontrar outras associações. Outra limitação é inerente ao próprio desenho do estudo, ou seja, a causalidade reversa.

A interface entre os determinantes sociais e os aspectos relacionados à obesidade grave são explorados neste trabalho mostrando que uma maior renda familiar per capita se associou à ausência de cardiopatia e maior consumo de vegetais folhosos e doces. Já a escolaridade não se manteve associada às condições de saúde e nutrição. Há poucos estudos sobre as condições de saúde e nutrição de obesos graves e suas inter -relações com os níveis de renda e escolaridade, assim esta pesquisa traz informações relevantes sobre esse grupo populacional e que auxiliam no aprofundamento do conhecimento desse tema. Essas informações podem ser úteis para direcionar o tratamento clínico e nutricional, e ainda como subsídio para elaborar protocolos que atendam às características peculiares dos obesos

\section{Referências}

1. Bessesen DH. Update on obesity. J Clin Endocrinol Metab 2008; 93(6):2027-2034.

2. Wanderley EN, Ferreira VA. Obesidade: uma perspectiva plural. Cien Saude Colet 2010; 15(1):185-194.

3. Flegal KM, Carroll MD, Ogden CL, Curtin LR. Prevalence and trends in obesity among US adults, 19992008. JAMA 2010; 303(3):235-241.

4. Instituto Brasileiro de Geografia e Estatística (IBGE). Coordenação de Trabalho e Rendimento. Pesquisa de orçamentos familiares, 2008-2009. Rio de Janeiro: IBGE 2010.

5. Flegal KM, Graubard BI, Williamson DF, Gail MH. Excess deaths associated with underweight, overweight, and obesity. JAMA 2005; 293(15):1861-1867.

6. Stommel M, Schoenborn CA. Variations in BMI and prevalence of health risks in diverse racial and ethnic populations. Obesity (Silver Spring) 2010; 18(9):18211826 graves. Conhecer melhor as características desse grupo pode contribuir com as equipes de saúde para que estejam mais preparadas para receber esses pacientes e, consequentemente, melhorar a adesão e a efetividade do tratamento. Poderão auxiliar também os gestores em saúde na elaboração de ações de promoção à saúde e políticas de saúde publica para combater e controlar a obesidade grave.

\section{Colaboradores}

APS Rodrigues trabalhou na coleta, análise dos dados, revisão de literatura e redação final. EA Silveira trabalhou na elaboração do projeto de pesquisa, concepção do artigo, metodologia, redação e aprovação da versão final.

\section{Agradecimentos}

Agradecemos à Coordenação de Aperfeiçoamento de Pessoal de Nível Superior (CAPES) pela bolsa de mestrado da autora principal, à graduanda em Nutrição Ana Gabriela Estavam Brito pelo auxílio na coleta dos dados, à Universidade Federal de Goiás pela bolsa de extensão da estagiária Ana Gabriela e aos pacientes do Ambulatório de Nutrição e Obesidade (ANOG) do Hospital das Clínicas/ UFG pela colaboração.

7. Ogden CL, Lamb MM, Carroll MD, Flegal KM. Obesity and socioeconomic status in adults: United States, 2005-2008. NCHS Data Brief 2010; (50):1-8.

8. Monteiro CA, Moura EC, Conde WL, Popkin BM. Socioeconomic status and obesity in adult populations of developing countries: a review. Bull World Health Organ 2004; 82(12):940-946.

9. Monteiro CA, Conde WL, Popkin BM. Independent effects of income and education on the risk of obesity in the Brazilian adult population. J Nutr 2001; 131(3):881S-886S

10. Thompson WG, Cook DA, Clark MM, Bardia A, Levine JA. Treatment of obesity. Mayo Clin Proc 2007; 82(1):93-101; quiz 101-102.

11. Residori L, Garcia-Lorda P, Flancbaum L, Pi-Sunyer FX, Laferrere B. Prevalence of co-morbidities in obese patients before bariatric surgery: effect of race. Obes Surg 2003; 13(3):333-340 
12. Merkus MP, Mathus-Vliegen LM, Broekhoff C, Heijnen AM. Extreme obesity: sociodemographic, familial and behavioural correlates in The Netherlands. J Epidemiol Community Health 1995; 49(1):22-27.

13. Howard NJ, Taylor AW, Gill TK, Chittleborough CR. Severe obesity: Investigating the socio-demographics within the extremes of body mass index. Obes Res Clin Pract 2008; 2(1):51-59.

14. Canedo FC, Silveira EA. Implementação do núcleo de atenção nutricional e pesquisa em obesidade no Hospital das Clínicas da UFG [dissertação]. Faculdade de Nutrição: Universidade Federal de Goiás; 2008.

15. Araújo CLP. Referências Antropométricas. In: Kac G, Sichieri R, Gigante DP, editors. Epidemiologia Nutricional. Rio de Janeiro: Fiocruz, Editora Atheneu; 2007. p. 65-77.

16. Block G, Hartman AM, Dresser CM, Carroll MD, Gannon J, Gardner L. A data-based approach to diet questionnaire design and testing. Am J Epidemiol 1986; 124(3):453-469.

17. World Health Organization (WHO). Global recommendations on physical activity for health. Geneva: WHO; 2010.

18. World Health Organization (WHO). Physical status: the use and interpretation of anthropometry. Report of a WHO Expert Committee. Geneva: WHO Technical Report; 1995.

19. World Health Organization (WHO). Obesity: preventing and managing the global epidemic. Report of a WHO consultation. Geneva: WHO Technical Report; 2000.

20. American Diabetes Association. Diagnosis and classification of diabetes melito. Diabetes Care 2011; 34(Supl. 1):S62-S69.

21. Sposito AC, Caramelli B, Fonseca FAH, Bertolami MC, Rassi Junior A, Comitê de Aterosclerose. IV Diretriz Brasileira sobre Dislipidemias e Prevenção da Aterosclerose: Departamento de Aterosclerose da Sociedade Brasileira de Cardiologia. Arq Bras Cardiol 2007; 88(Supl. 1):2-19.

22. Fisbach F. Manual de enfermagem: exames laboratoriais e diagnósticos. Rio de Janeiro: Guanabara Koogan; 2002.

23. Porto MCV, Brito IC, Calfa ADF, Amoras M, Villela NB, Araújo LMB. Perfil do obeso classe III do ambulatório de obesidade de um hospital universitário de Salvador, Bahia. Arquivos Brasileiros de Endocrinologia \& Metabologia 2002; 46(6):668-673.

24. Costa ACC, Ivo ML, Cantero WdB, Tognini JRF. Obesidade em pacientes candidatos a cirurgia bariátrica. Acta Paulista de Enfermagem 2009; 22(1):55-59.

25. Lima LP, Sampaio HAC. Caracterização socioeconômica, antropométrica e alimentar de obesos graves. Cien Saude Colet 2007; 12(4):1011-1020.

26. Buchwald H, Avidor Y, Braunwald E, Jensen MD, Pories W, Fahrbach K, Schoelles K. Bariatric surgery: a systematic review and meta-analysis. JAMA 2004; 292(14):1724-1737.
27. Lins APM, Sichieri R, Coutinho WF, Ramos EG, Peixoto MVM, Fonseca VM. Alimentação saudável, escolaridade e excesso de peso entre mulheres de baixa renda. Cien Saude Colet 2013; 18(2):357-366.

28. Jakobsen GS, Hofso D, Roislien J, Sandbu R, Hjelmesaeth J. Morbidly obese patients--who undergoes bariatric surgery? Obes Surg 2010; 20(8):1142-1148.

29. Travassos C, Viacava F, Pinheiro R, Brito A. Utilização dos serviços de saúde no Brasil: gênero, características familiares e condição social. Rev Panam Salud Pública 2002; 11(5-6):365-373.

30. Gigante DP, Dias-da-Costa JS, Olinto MTA, Menezes AMB, Silvia M. Obesidade da população adulta de Pelotas, Rio Grande do Sul, Brasil e associação com nível sócio-econômico. Cad Saude Publica 2006; 22(9):18731879.

31. Molarius A, Seidell JC, Sans S, Tuomilehto J, Kuulasmaa K. Educational level, relative body weight, and changes in their association over 10 years: an international perspective from the WHO MONICA Project. Am J Public Health 2000; 90(8):1260-1268.

32. Stamler J, Elliott P, Appel L, Chan Q, Buzzard M, Dennis B, Dyer AR, Elmer P, Greenland P, Jones D, Kesteloot H, Kuller L, Labarthe D, Liu K, Moag-Stahlberg A, Nichaman M, Okayama A, Okuda N, Robertson C, Rodriguez B, Stevens M, Ueshima H, Horn LV, Zhou B. Higher blood pressure in middle-aged American adults with less education-role of multiple dietary factors: the INTERMAP study. J Hum Hypertens 2003; 17(9):655775.

33. Vargas CM, Ingram DD, Gillum RF. Incidence of hypertension and educational attainment: the NHANES I epidemiologic followup study. First National Health and Nutrition Examination Survey. Am J Epidemiol 2000; 152(3):272-278.

34. Clark AM, DesMeules M, Luo W, Duncan AS, Wielgosz A. Socioeconomic status and cardiovascular disease: risks and implications for care. Nat Rev Cardiol 2009; 6(11):712-722.

35. Lee DS, Chiu M, Manuel DG, Tu K, Wang X, Austin PC, Mattern MY, Mitiku TF, Svenson LW, Putnam W, Flanagan WM, Tu JV; Canadian Cardiovascular Outcomes Research Team. Trends in risk factors for cardiovascular disease in Canada: temporal, socio-demographic and geographic factors. CMAJ 2009; 181(3-4):E55-E66.

36. Instituto Brasileiro de Geografia e Estatística (IBGE). Pesquisa de orçamentos familiares 2002-2003. Rio de Janeiro: IBGE; 2004.

Artigo apresentado em 06/10/2013

Aprovado em 18/03/2014

Versão final apresentada em 24/03/2014 